\title{
The interplay between abdominal aortic aneurysm, metabolic syndrome and fatty liver disease: a retrospective case-control study
}

This article was published in the following Dove Press journal: Diabetes, Metabolic Syndrome and Obesity: Targets and Therapy

\author{
Mahmud Mahamid ${ }^{1,2}$ \\ Tawfik Khoury 2,3 \\ Baker Mahamid' \\ Wisam Sbeit ${ }^{2,3}$ \\ Amir Mari ${ }^{1}$ \\ Wiliam Nseir ${ }^{2,4}$ \\ 'Gastroenterology and Hepatology Unit, \\ Nazareth Hospital EMMS, Nazareth, \\ Israel; ${ }^{2}$ Faculty of Medicine in the Galilee, \\ Bar-Ilan University, Safed, Israel; \\ ${ }^{3}$ Department of Gastroenterology, \\ Galilee Medical Center, Nahariya, Israel; \\ ${ }^{4}$ Internal Medicine Department, Nazareth \\ Hospital EMMS, Nazareth, Israel
}

Correspondence: Mahmud Mahamid Division of Internal Medicine, Gastroenterology and Endoscopy Unit, EMMS, the Nazareth Hospital, Nazareth 16100 , Israel

Tel +972 4602885 I

Fax +97274755 905।

Emailmahmudmahamid@yahoo.com
Background: Abdominal aortic aneurysm (AAA) and fatty liver disease are both associated with the metabolic syndrome (MS); the aim of this study was to investigate whether patients with AAA are also at a higher risk for fatty liver disease.

Methods: A case-control retrospective study. Patients diagnosed with AAA were compared with age- and sex-matched controls regarding the prevalence of fatty liver disease. Extracted data include anthropometric parameters, clinical and laboratory data, and liver imaging.

Results: 995 patients were enrolled in the final analysis, 495 patients with AAA and 500 age- and sex-matched controls. The prevalence of fatty liver disease among AAA subjects was $48.9 \%$ compared with $21.2 \%$ among the controls $(P<0.005)$. After adjusting for age, smoking, body mass index, and MS components, the logistic regression analysis indicates that AAA (men: OR 1.29, 95\% CI 1.17, 1.49, $P=0.001$; women: OR $1.23,95 \%$ CI $1.06,1.43$, $P=0.002$ ), obesity (men: OR $1.32,95 \%$ CI $1.17,1.59, P<0.001$; women: OR $1.32,95 \%$ CI $1.07,1.52, P=0.012$ ), hypertension (men: OR $1.23,95 \%$ CI $1.13,1.46, P=0.001$; women: OR 1.13, 95\% CI 1.00, 1.33, $P=0.045$ ), MS (men: OR 1.31, 95\% CI 1.19, 1.53, $P=0.001$; women: OR 1.28, 95\% CI 1.16, 1.42, $P=0.002$ ) were associated with non-alcoholic fatty liver disease/non-alcoholic steatohepatitis (NAFLD/NASH). The prevalence of liver cirrhosis was $1.23 \%$; subjects with obesity, diabetes, hypertension, and AAA had increased risk for cirrhosis (OR 1.89, 95\% CI 1.18, 3.22, $P=0.014$; OR 1.27, 95\% CI 1.09, 2.72, $P=0.0027$; OR 2.08, 95\% CI 1.29, 3.42, $P=0.004$; OR 1.73, 95\% CI 1.08, 2.87, $P=0.027$, respectively).

Conclusion: AAA patients are at increased risk for NAFLD/NASH, may predict advance liver disease and liver cirrhosis.

Keywords: fatty liver, abdominal aortic aneurysm, metabolic syndrome

\section{Introduction}

The aorta is the largest blood vessel in the body that supplies blood from the left ventricle of the heart to the systemic vasculature of the body. Adults have three layers and its diameter ranges according to its different parts: $3 \mathrm{~cm}$ ascending arch of aorta, $2.5 \mathrm{~cm}$ thoracic and 1.8-2 cm descending aorta. ${ }^{1,2}$ Abdominal aortic aneurysms (AAA) represent an important public health problem with prevalence between $1.3 \%$ and $12.5 \%$ in males and between $0.0 \%$ and $5.2 \%$ in females. ${ }^{3}$ AAA is defined as an enlargement in abdominal aortic diameter of at least 1.5 times the original diameter, which is $2 \mathrm{~cm}$, thus the value of $>3 \mathrm{~cm}$ indicates AAA. ${ }^{1,4}$ A "true aneurysm" must include must three layers of the artery such as intima, media, and adventitia. 
AAA is the 14th leading cause of death in the United States. Annually, there are about 9000 death incidences as a result of AAA rupturing and around 1400 death incidences as a result of aiming to prevent the rupture of AAA. AAA is divided into the upper and lower parts in relation to the renal arteries' location; $80 \%$ of AAA is located below the renal arteries. ${ }^{2,3,5,6}$ Risk factors include: male gender, Caucasian, history of smoking (approximately more than 100 cigarettes in a lifetime) and above the age of 65. Other risk factors associated with AAA include family history of AAA, acute coronary syndrome, peripheral artery disease, hypercholesterolemia, hypertension, cerebrovascular diseases and short stature, atherosclerosis, and peripheral vascular diseases. ${ }^{7-9}$

Most AAA's carry no signs or symptoms and are mainly detected accidentally by radiological examinations such as magnetic resonance imaging, computed tomography and ultrasound that are conducted for alternative aims. ${ }^{10}$

The modality of choice for screening for AAA is abdominal ultrasound which is both inexpensive and safe and has a 95-100\% sensitivity and almost $100 \%$ specificity. ${ }^{7,11}$ The prevalence of AAA globally ranges between $1 \%$ and $2 \%$ while the diagnosis made by abdominal ultrasound among the elderly population $(65+)$ is $5.5 \%$ in males and $1 \%$ in females. AAA is known to increase with age and can reach up to $9 \%$ in elderly people with multiple risk factors. The prevalence of AAA $>5 \mathrm{~cm}$ in diameter in males at ages 50-79 is estimated at around $0.5 \%{ }^{3,5-7}$

Studies have shown that screening once for AAA is sufficient if the result is negative. ${ }^{6}$ The balance between incidence and cost-effectiveness for AAA screening in asymptomatic patients, combined with increased numbers of elective aneurysm surgery may reduce the mortality rate. ${ }^{12-14}$ Since the prevalence of AAA in the general population is low (1-2\%), targeting specific high-risk populations is recommended. ${ }^{5,6}$ Indications for AAA screening according to "The Society for Vascular Surgery and The Society for Vascular Medicine and Biology" are: male $>60-85$ years of age, female $>60-85$ year of age with cardiovascular risk factors, and both men and women $>50$ years of age with family history of AAA. ${ }^{7}$ According to previous research, early screening for AAA reduces its mortality by $42 \%{ }^{4}$ Moreover, imaging study performing for AAA screening can reveal additional information regarding other intra-abdominal organs including liver. ${ }^{15}$

Non-alcoholic fatty liver disease (NAFLD) is an important global public health abnormality and the most common cause of chronic liver disease globally. ${ }^{16}$ NAFLD is closely correlated with simple hepatic steatosis and an excess of intrahepatic ectopic triglyceride deposition. ${ }^{16}$ NAFLD may progress to non-alcoholic steatohepatitis (NASH) and advanced liver fibrosis, cirrhosis, and hepatocellular carcinoma. ${ }^{16,17}$ NAFLD is often identified incidentally by the finding of elevated liver enzymes. Statistics show that the occurrence of NASH in Europe and USA ranges from $14 \%$ to $20 \%$; this increase in the prevalence is directly related to the obesity epidemic seen in these populations. $^{16,17}$

NAFLD is often referred to as the hepatic manifestation of the metabolic syndrome (MS). With the marked increase in obesity, the associated rise of NASH/NAFLD and MS are a major public health concern. Diabetes mellitus (DM), obesity, and hyperlipidemia are also common components of MS, which is frequently associated with NAFLD ${ }^{16}$ and also are considered as risk factors for AAA. ${ }^{17}$

According to recently published studies, the overall mortality and morbidity among NASH/NAFLD patients is higher compared to the general population; resulting from increases in cardiovascular diseases, malignancies and liver-related mortality. ${ }^{18}$

Interestingly, recent medical literature supports the hypothesis that NAFLD is a multisystem disease involving variety of extra-hepatic organs and systems. There are strong associations and links between NAFLD and cardiovascular disease, as well as metabolic disorders such as diabetes, endothelial dysfunction and obesity. Moreover, fatty liver disease is associated with colorectal cancer, hypothyroidism, polycystic ovarian syndrome, obstructive sleep apnea, osteoporosis, and iron overload..$^{19,21}$

NAFLD patients are at increased risk for cardiovascular disorders, including hypertension, atherosclerosis, endothelial dysfunction, carotid intima-media thickness, impaired flow-mediated vasodilatation and arterial stiffness, atrial fibrillation insulin resistance, dyslipidemia ischemic heart disease and aortic valve sclerosis. ${ }^{20,21}$ The aim of the current study, therefore, is to evaluate whether NAFLD/NASH is an independent risk factor for the development of AAA.

\section{Materials and methods}

The current study received ethical approval from the local hospital ethical committee and was conducted according to the Helsinki declaration and its subsequent amendments. Data were coded in order to preserve the anonymity of the patients. Informed consent was waived because of the noninterventional study design. 


\section{Patient's selection}

We conducted a case-control retrospective study, using the databases of the Nazareth Hospital, EMMS (Nazareth, Israel). All patients diagnosed with AAA (by ultrasound, computed tomography or/and aortic angiography) who were diagnosed, followed or admitted to the hospital between 2010 and 2018 and who underwent abdominal imaging (abdominal ultrasound and/or abdominal computer tomography) were considered potentially eligible and enrolled in the study regardless of their clinical conditions or the cause for performing the abdominal imaging, as well as their age- and sex-matched controls .

AAA is defined as an enlargement in abdominal aortic diameter of at least 1.5 times the original diameter, which is $2 \mathrm{~cm}$, thus the value of $>3 \mathrm{~cm}$ indicates AAA.

Patients with other hepatic pathology or autoimmune markers were excluded (such as alcoholic liver disease, druginduced liver injury, autoimmune hepatitis, viral hepatitis, cholestatic liver diseases and metabolic/genetic liver disease) as assessed by clinical, laboratory, radiological and/or histological criteria/tests (serology of viral hepatitis A, B and C, autoimmune markers including ANA, anti-LKM, anti-smooth mussels, protein electrophoresis, immunoelectrophoresis, metabolic markers such serum ceruloplasmin, $24 \mathrm{hrs}$ urine collection for copper, ferritin, iron, transferrin saturation, thyroid-stimulating hormone (TSH), HbAlc and alpha-1 antitrypsin).

Extracted data included demographic variables (age, gender), body mass index (BMI), laboratory parameters as: alanine transaminase (ALT), aspartate transaminase (AST), lowdensity lipoprotein cholesterol (LDL), high-density lipoprotein cholesterol, triglycerides, glycated hemoglobin (hemoglobin Alc or HbAlc), and C-reactive protein (CRP), and the results of abdominal ultrasound and/or abdominal computer tomography performing in the study period .

\section{Statistical analysis}

Before commencing any statistical processing and analysis, data were visually inspected and checked for outliers. A logistic regression model was carried out to evaluate the NAFLD/NASH as independent risk factors for AAA.

Continuous variables were computed as mean $\pm \mathrm{SD}$, whereas categorical variables were expressed as percentages. Univariate and multivariate regression analyses were carried out using AAA as dependent variables. All statistical analyses were performed with the commercial software Statistical Package for Social Science (SPSS version 24.0,
IBM, Chicago, IL, USA). ROC analysis was performed with the commercial software MedCalc Statistical Software version 17.9.7 (MedCalc Software bvba, Ostend, Belgium; http://www.medcalc.org; 2017). Figures with $P<0.05$ were considered statistically significant.

\section{Results}

Overall 995 patients were enrolled in the final analysis, 495 patients with AAA and 500 age- and sex-matched controls. 621 patients with documented AAA were initially enrolled in this study. Subjects missing imaging information regarding the liver $(n=68)$, those with history or evidence of other liver disease than NAFLD/NASH $(\mathrm{n}=45)$, and those with indefinite diagnosis of NAFLD/ NASH $(n=13)$ were excluded.

Among the AAA group [321 (65\%) were males and 174 (35\%) females]. In the control group, 321 subjects $(64.2 \%)$ were males. The mean age was $68.43 \pm 12.71$ years among the AAA group and $67.92 \pm 11.84$ years among the control group. The mean BMI was $25 \pm 3.12$ among the AAA group and $25 \pm 4.26$ among the control group. The prevalence of fatty liver disease NASH/NAFLD among AAA subjects was $48.9 \%(\mathrm{n}=242)(42.3 \%$ among women and $56.4 \%$ among men) and 106 subjects (21.1\%) among the control group $(P<0.005)$. The baseline characteristics of the participants from both groups are presented in Table 1. Among the AAA group, the baseline characteristics of those with and without NASH/NAFLD are presented in Table 2.

Table I The baseline characteristics of AAA patients and control group

\begin{tabular}{|l|l|l|l|}
\hline & $\begin{array}{l}\text { AAA } \\
\text { group }\end{array}$ & $\begin{array}{l}\text { Control } \\
\text { group }\end{array}$ & P-value \\
\hline Number of patients & 495 & 500 & \\
Mean age \pm SD & $68.43 \pm 12.7$ & $67.92 \pm I 1.84$ & NS \\
Sex \% (males) & $65 \%$ & $64.2 \%$ & NS \\
BMI & $25.3 \pm 3.12$ & $25 \pm 4.26$ & NS \\
Fatty liver per imaging (\%) & $242(48.9 \%)$ & $106(21.1 \%)$ & $<0.005$ \\
TG (mg/dL) & $151.1 \pm 47.8$ & $149.9 \pm 29$ & NS \\
HDL (mg/dL) & $34 \pm 8.2$ & $35.3 \pm 7.6$ & NS \\
LDL (mg/dL) & $148.2 \pm 28.5$ & $147.7 \pm 25.3$ & NS \\
CRP (mg/L) & $2.1 \pm 2.6$ & $1.6 \pm 3.7$ & NS \\
Smoking (\%) & $28.3 \%$ & $26.5 \%$ & NS \\
HbAIC (\%) & $5.6 \pm 0.7$ & $5.7 \pm 0.9$ & NS \\
HOMA-IR & $3.19 \pm 1$ & $2.92 \pm 1.4$ & NS \\
Hypertension (\%) & $17.4 \%$ & $16.9 \%$ & NS \\
Hyperlipidemia (\%) & $32.7 \%$ & $31.9 \%$ & NS \\
\hline
\end{tabular}

Abbreviations: AAA, abdominal aorta aneurysm; BMl, body mass index; TG, triglycerides; HDL, high-density lipoprotein; LDL, low-density lipoprotein; CRP, Creactive protein. 
Table 2 Baseline characteristics of abdominal aorta aneurysm subjects with and without fatty liver

\begin{tabular}{|c|c|c|c|c|}
\hline Variable & Total $(n=495)$ & With NAFLD $(n=242)$ & Without NAFLD $(n=253)$ & $P$-value \\
\hline Male sex & $321(65)$ & $168(69.4)$ & $159(62.8)$ & 0.056 \\
\hline Age, years & $68.4 \pm 11.7$ & $63.7 \pm 9.9$ & $66.2 \pm 10.0$ & $<0.05$ \\
\hline $\mathrm{BMI}, \mathrm{kg} / \mathrm{m}^{2}$ & $25 \pm 3.2$ & $29.2 \pm 4.3$ & $26.1 \pm 4.1$ & $<0.05$ \\
\hline Hypertension (\%) & $231(50.3)$ & $134(55.3)$ & $104(47)$ & $<0.05$ \\
\hline Diabetes (\%) & $135(33.3)$ & 91 (36.8) & $74(34.1)$ & $<0.05$ \\
\hline Smoking (\%) & $98(21.3)$ & $64(26.4)$ & $48(22.2)$ & $<0.05$ \\
\hline Glycated hemoglobin, \% & $5.1 \pm 0.6$ & $5.9 \pm 0.8$ & $5.5 \pm 0.4$ & $<0.05$ \\
\hline Triglyceride, mg/dL & $93.4 \pm 63.2$ & $99.6 \pm 69.2$ & $92.1 \pm 60.8$ & $<0.05$ \\
\hline CRP (mg/L) & $1.7 \pm 3.8$ & $5.1 \pm 2.6$ & $3.1 \pm 1.6$ & $<0.05$ \\
\hline $\mathrm{HDL}-\mathrm{C}, \mathrm{mg} / \mathrm{dL}$ & $53.2 \pm 13.1$ & $49.8 \pm 11.6$ & $55.5 \pm 13.4$ & $<0.05$ \\
\hline LDL-C, mg/dL & $|20.2 \pm 3| . \mid$ & $|32.9 \pm 4| . \mid$ & $1|9.9 \pm 4| .1$ & $<0.05$ \\
\hline AST, U/L & $32.9 \pm 12.1$ & $67 \pm 31.2$ & $38.2 \pm \mid 7.1$ & $<0.05$ \\
\hline ALT, U/L & $36.3 \pm 10.2$ & $51.3 \pm 25.2$ & $41.3 \pm 22.2$ & $<0.05$ \\
\hline Metabolic syndrome & $48(10.9)$ & $39(16.1)$ & $22(10.1)$ & $<0.05$ \\
\hline
\end{tabular}

Abbreviations: NAFLD, non-alcoholic fatty liver disease; BMI, body mass index; CRP, C-reactive protein; HDL, high-density lipoprotein cholesterol; LDL, low-density lipoprotein cholesterol; ALT, alanine transaminase; AST, aspartate transaminase.

Regarding the AAA group, when comparing male patients with NASH/NAFLD with those without NASH/ NAFLD, they were younger, had higher BMI, higher prevalence of hypertension, diabetes, and smoking, as well as higher levels of ALT, AST, LDL, triglycerides, HbA1c, and CRP. Female AAA patients had higher levels of total cholesterol, triglycerides, and ALT. Moreover, patients with NASH/NAFLD, from both genders, had a higher prevalence of MS as well as its components; obesity, hyperlipidemia, hypertension, and diabetes $(P<0.001)$. The prevalence of NASH/NAFLD according to the MS components is presented in Table 3.

Regarding the relationship between NASH/NAFLD and AAA, the adjusted ORs for NASH/NAFLD in both genders were calculated. After adjusting for age, smoking, BMI, and MS components, the logistic regression analysis indicates that AAA (men: OR 1.29, 95\% CI 1.17, 1.49, $P=0.001$; women: OR $1.23,95 \%$ CI $1.06,1.43, P=0.002$ ), obesity (men: OR 1.32, 95\% CI 1.17, 1.59, $P<0.001$; women: OR 1.32, 95\% CI 1.07, 1.52, $P=0.012$ ), hypertension (men: OR $1.23,95 \%$ CI 1.13, 1.46, $P=0.001$; women: OR $1.13,95 \%$ CI $1.00,1.33, P=0.045$ ), MS (men: OR $1.31,95 \%$ CI 1.19, 1.53, $P=0.001$; women: OR $1.28,95 \%$ CI 1.16, 1.42, $P=0.002$ ) were associated with NAFLD/ NASH. Whereas diabetes and smoking were only associated with NASH/NAFLD in men (OR $1.27,95 \%$ CI $1.07,1.52, P=0.010$; and OR $1.26,95 \%$ CI $1.22,1.62$, $P<0.001)$. Hyperlipidemia was not an independent sigificant risk factor for the increase prevalence of NAFLD/NASH in both genders $(P>0.05)$.
Regarding the association of the specific imaging characteristics of liver cirrhosis (nodular border and splenomegaly) associated with ANFLD/NASH and AAA, among the participants with NAFLD/NASH, the imaging prevalence of cirrhosis was $1.23 \%(n=3)[0.4 \%(n=1)$ among females, and $0.8 \%(n=2)$ among men]. When we evaluated the relationship between the imaging characteristics associated with cirrhosis with MS components (including obesity, diabetes hypertension, and hyperlipidemia) and AAA, among men, the prevalence of cirrhosis was significantly

Table 3 Prevalence of NASH/NAFLD (\%) according to the status of metabolic syndrome components and abdominal aortic aneurysm

\begin{tabular}{|l|l|l|}
\hline Variable & Men & Women \\
\hline Met-S & 66.5 & 47.2 \\
Without Met-S & 51.2 & 38.6 \\
P-value & $<0.00 \mathrm{I}$ & $<0.00 \mathrm{I}$ \\
Obesity & $65 . \mathrm{I}$ & 45.3 \\
Without obesity & $54 . \mathrm{I}$ & 41.2 \\
P-value & $<0.00 \mathrm{I}$ & $<0.00 \mathrm{I}$ \\
Diabetes & 67.3 & 49.8 \\
Without diabetes & 53.8 & 38.9 \\
$P$-value & $<0.00 \mathrm{I}$ & $<0.00 \mathrm{I}$ \\
Hyperlipidaemia & 63.8 & 44.3 \\
Without hyperlipidaemia & 51.3 & 39.4 \\
P-value & $<0.00 \mathrm{I}$ & $<0.00 \mathrm{I}$ \\
Hypertension & 65.6 & 46.3 \\
Without hypertension & 48.5 & 37.6 \\
$P$-value & $<0.00 \mathrm{I}$ & $<0.00 \mathrm{I}$ \\
\hline
\end{tabular}

Abbreviation: NASH/NAFLD, non-alcoholic steatohepatitis/non-alcoholic fatty liver disease. 
increased in subjects with obesity, diabetes, hypertension and AAA (all $P<0.01$ ); no significant differences were found among women with NASH/NAFLD Table 4.

After calculating the adjusted ORs for these imaging characteristics associated with cirrhosis. we found that men with obesity, diabetes, hypertension, and AAA had increased risk for cirrhosis (OR 1.89, 95\% CI 1.18, 3.22, $P=0.014$; OR 1.27, 95\% CI 1.09, 2.72, $P=0.0027$; OR $2.08,95 \%$ CI $1.29,3.42, P=0.004$; OR $1.73,95 \%$ CI $1.08,2.87, P=0.027$, respectively).

\section{Discussion}

NAFLD is an important global public health challenge and the most common cause of chronic liver disease universally. ${ }^{16}$ NAFLD is closely associated with simple hepatic steatosis and excess of intrahepatic ectopic triglyceride deposition. ${ }^{16}$ NAFLD is generally considered as the hepatic manifestation of the MS. Primary care providers are noticing a major increase in obesity, NASH/NAFLD and MS all of which are now a major public health concern. ${ }^{17} \mathrm{DM}$, obesity, and hyperlipidemia are also common components of MS, which is frequently associated with NAFLD. ${ }^{21}$ Previous meta-analysis showed the role of risk factors such as gender, smoking, atherosclerosis, hypertension, obesity, and peripheral vascular disease in the development of AAA. ${ }^{5}$ MS which is composed of several of these risk factors are also highly correlated with fatty liver disease. ${ }^{21}$ We conducted a case-control study to better define the possible correlation between AAA and fatty liver disease. ${ }^{15,16}$

Our study was designed to assess the possible association between ANFLD/NASH and AAA as well as other components of MS that can be found among patients with AAA.

As noted, previously published data reported that AAA is closely related to several components of the $\mathrm{MS}^{3}$ which is confirmed in our current study of patients with AAA.
The majority are elderly men, have a high BMI, and high prevalence of hypertension, diabetes, hyperlipidemia, and smoking. Higher prevalence of those risk factors associated with AAA was seen among patients suffering from both AAA and NAFLD/NASH across both genders.

Given the fact that AAA subjects with NAFLD/NASH had worse MS parameters with higher MS prevalence, we evaluated the adjusted ORs for NAFLD/NASH. After adjustment, logistic regression analysis revealed that patients with AAA were at high risk for NAFLD/NASH (men: OR 1.29, 95\% CI 1.17, 1.49, $P=0.001$; women: OR 1.23, 95\% CI 1.06, 1.43, $P=0.002$ ), obesity (men: OR $1.32,95 \%$ CI $1.17,1.59, P<0.001$; women: OR 1.32, 95\% CI 1.07, 1.52, $P=0.012$ ), hypertension (men: OR 1.23, 95\% CI 1.13, 1.46, $P=0.001$; women: OR 1.13, 95\% CI 1.00, 1.33, $P=0.045$ ), MS (men: OR 1.31, 95\% CI 1.19,1.53, $P=0.001$; women: OR $1.28,95 \%$ CI 1.16 , 1.42, $P=0.002$ ). Diabetes and smoking were only associated with NASH/NAFLD in men (OR 1.27, 95\% CI 1.07, 1.52, $P=0.010$; and OR 1.26, 95\% CI 1.22, 1.62, $P<0.001)$. Hyperlipidemia was not an independent significant risk factor for the increase prevalence of NAFLD/ $\mathrm{NASH}$ in both genders.

To our knowledge, there are no data in the medical literature regarding the association between AAA and NAFLD/NASH. In our study the prevalence of NAFLD/ $\mathrm{NASH}$ among AAA is $48.9 \%$ (42.3\% among females and $56.4 \%$ among men). Furthermore, this association persisted even after full adjustments.

Our study identified an association of liver cirrhosis seen on non-invasive imaging among NAFLD/NASH subjects and AAA, with a prevalence of $1.23 \%$. Among men, the prevalence of cirrhosis was significantly increased in subjects with obesity, diabetes, hypertension and AAA (all $P<0.01$ ); no significant differences were found among women with NASH/NAFLD.

Table 4 The adjusted OR for NAFLD/NASH in both genders by abdominal aorta aneurysm, obesity, hypertension, metabolic syndrome diabetes, and smoking

\begin{tabular}{|l|l|l|l|l|l|l|}
\hline Variable & Men OR & Men CI & P-value & Women OR & Women Cl & $P$-value \\
\hline AAA & 1.29 & $1.17-1.49$ & 0.001 & 1.23 & $1.06-1.43$ & 0.002 \\
Obesity & 1.32 & $1.17-1.59$ & $<0.001$ & 1.32 & $1.07-1.52$ & 0.012 \\
Hypertension & 1.23 & $1.13-1.46$ & 0.001 & 1.13 & $1.00-1.33$ & 0.045 \\
Metabolic syndrome & 1.31 & $1.19-1.53$ & 0.001 & 1.28 & $1.16-1.42$ & 0.027 \\
Diabetes & 1.27 & $1.07-1.52$ & 0.010 & 1.01 & $1.07-1.18$ & 0.052 \\
Smoking & 1.26 & $1.22-1.62$ & $<0.001$ & 1.08 & $1.02-1.13$ & 0.061 \\
\hline
\end{tabular}

Abbreviations: NASH/NAFLD, non-alcoholic steatohepatitis/non-alcoholic fatty liver disease; AAA, abdominal aortic aneurysm. 
After calculating the adjusted ORs for imaging features of cirrhosis, we found that men with AAA obesity, diabetes, and hypertension had a significantly increased risk for cirrhosis (OR 1.89, 95\% CI 1.18, 3.22, $P=0.014$; OR $1.27,95 \%$ CI $1.09,2.72, P=0.0027$; OR $2.08,95 \%$ CI $1.29,3.42, P=0.004 ;$ OR $1.73,95 \%$ CI $1.08,2.87$, $P=0.027$, respectively). These findings suggest that AAA can be associated not only with the presence of NAFLD/ NASH but can also predict the advance stage of fatty liver disease and transformation to liver cirrhosis. Visible hallmark of AAA pathogenesis includes inflammation, smooth muscle cell apoptosis, extracellular matrix degradation and oxidative stress. These pathological processes were widely studied in NASH and MS and assumed to represent the common pathway and the interplay between AAA, NASH and MS. ${ }^{1,17}$

Our study has several limitations, the main limitation of our study is the retrospective and case-control design with the drawback of selection bias, which afflicts all case-control studies of this kind. Hence, we were unable to reassess diagnosis and prevalence of NAFLD/NASH on a later time, thus follow-up and natural history learning were unfeasible. Second, our data are based on the end result of the diagnostic/therapeutic process performed by the EMMS hospital specialist in gastroenterology, vascular surgery as well as interventional/ diagnostic radiology and information with regard to the exact criteria and the gold standard criteria used to define the AAA, and NAFLD/NASH as well as MS are lacking as liver biopsy and/or aortic angiography was not performed in the majority of patients. Finally, self-reported viral hepatitis, and/or other liver diseases may have introduced recall bias.

In conclusion, patients with AAA and the MS have an increased risk of NAFLD/NASH in both genders, in men specifically, AAA, obesity, hypertension, and MS were significantly associated with NAFLD/NASH. Moreover, AAA, obesity, diabetes, and hypertension may also confer a higher risk for more advanced liver disease and liver cirrhosis among fatty liver disease patients.

The potential role of AAA and MS components in the development of liver cirrhosis remains to be elucidated by large prospective future studies.

\section{Ethics statement}

This study has been approved by the local ethics committee of the Nazareth EMMS Hospital.

\section{Acknowledgment}

We would like to thank our colleague, Moshe Rubin, MD, for his kind help with language editing.

\section{Disclosure}

This research did not receive any specific grant from funding agencies in the public, commercial, or not-forprofit sectors. The authors declare that there are no conflicts of interest in this work.

\section{References}

1. Bergqvist D, Björck M, Wanhainen A. Abdominal aortic aneurysm to screen or not to screen. Eur J Vasc Endovasc Surg. 2008;35(1):1318. doi:10.1016/j.ejvs.2007.06.012

2. Longo D, Fauci A, Kasper D, et al. Harrisons's principles of internal medicine, 18th ed. McGraw Hill: Graefe's archive for clinical and experimental ophthalmology. 2012;250(9):1407-1408.

3. Gillum R. Epidemiology of aortic aneurysm in the United States. J Clin Epidemiol. 1995;48(11):1289-1298. doi:10.1016/0895-4356(95)00045-3

4. Ouriel K, Green R, Donayre C, Shortell C, Elliott J, DeWeese J. An evaluation of new methods of expressing aortic aneurysm size: relationship to rupture. J Vasc Surg. 1992;15(1):12-20. doi:10.1016/ 0741-5214(92)70008-9

5. Lindholt J, Norman P. Screening for abdominal aortic aneurysm reduces overall mortality in men. a meta-analysis of the mid- and long-term effects of screening for abdominal aortic aneurysms. $J$ Vasc Surg. 2008;48(2):502. doi:10.1016/j.jvs.2008.06.025

6. Cueff C, Keenan N, Krapf L, et al. Screening for abdominal aortic aneurysm in coronary care unit patients with acute myocardial infarction using portable transthoracic echocardiography. Eur Heart J. 2011;13(7):574-578.

7. Fleming C, Whitlock EP, Beil TL, Lederle FA. Screening for abdominal aortic aneurysm: recommendations from the U.S. preventive services task force. Ann Intern Med. 2005;142(3):I-52.

8. Roshanali F, Mandegar M, Yousefnia M, Mohammadi A, Baharvand B. Abdominal aorta screening during transthoracic echocardiography. Echocardiography. 2007;24(7):685-688. doi:10.1111/j.15408175.2007.00467.x

9. Baumgartner I, Hirsch A, Abola M, et al. Cardiovascular risk profile and outcome of patients with abdominal aortic aneurysm in outpatients with atherothrombosis: data from the Reduction of Atherothrombosis for Continued Health (REACH) registry. J Vasc Surg. 2008;48(4):808-814. doi:10.1016/j.jvs.2008.05.026

10. Aronow W. Abdominal aortic aneurysm. J Clin Exp Cardiolog. 2014;05(09). doi:10.4172/2155-9880

11. LaRoy L, Cormier P, Matalon T, Patel S, Turner D, Silver B. Imaging of abdominal aortic aneurysms. Am J Roentgenol. 1989;152(4):785792. doi:10.2214/ajr.152.4.785

12. Navas E. Abdominal aortic aneurysm screening during transthoracic echocardiography: cardiologist and vascular medicine specialist interpretation. World J Cardiol. 2012;4(2):31. doi:10.4330/wjc.v4.i2.31

13. Giaconi S, Lattanzi F, Orsini E, Prosperi R, Tartarini G. Feasibility and accuracy of a rapid evaluation of the abdominal aorta during routine transthoracic echocardiography. Ital Heart J Suppl. 2003;4:332-336.

14. McPhee J, Hill J, Eslami M. The impact of gender on presentation, therapy, and mortality of abdominal aortic aneurysm in the United States, 2001-2004. J Vasc Surg. 2007;45(5):891-899. doi:10.1016/j. jvs.2007.01.043

15. Dupont A, Elkalioubie A, Juthier F, et al. Frequency of abdominal aortic aneurysm in patients undergoing coronary artery bypass grafting. $\mathrm{Am} \mathrm{J}$ Cardiol. 2010;105(11):1545-1548. doi:10.1016/j.amjcard.2010.01.011 
16. Younossi ZM, Koenig AB, Abdelatif D, Fazel Y, Henry L, Wymer M Global epidemiology of nonalcoholic fatty liver disease-meta-analytic assessment of prevalence, incidence, and outcomes. Hepatology. 2016;64(1):73-84. doi:10.1002/hep.28431

17. Milic S, Mikolasevic I, Krznaric-Zrnic I, et al. Nonalcoholic steatohepatitis: emerging targeted therapies to optimize treatment options. Drug Des Dev Ther. 2015;20(9):4835-4845. doi:10.2147/DDDT. S64877

18. Bertolotti M, Lonardo A, Mussi C, et al. Nonalcoholic fatty liver disease and aging: epidemiology to management. World $J$ Gastroenterol. 2014;20:14185-14204. doi:10.3748/wjg.v20.i39.14185
19. Mendonca FM, de Sousa FR, Barbosa AL, et al. Metabolic syndrome and risk of cancer: which link? Metabolism. 2015;64:182-189. doi:10.1016/j.metabol.2014.10.008

20. Chalasani N, Younossi Z, Lavine JE, et al. The diagnosis and management of non-alcoholic fatty liver disease: practice guideline by the American association for the study of liver diseases, American college of gastroenterology, and the American gastroenterological association. Hepatology. 2012;55(6):2005-2023. doi:10.1002/hep.25762

21. Clark JM, Diehl AM. Defining nonalcoholic fatty liver disease: implications for epidemiologic studies. Gastroenterology. 2003;124 (1):248-250. doi:10.1053/gast.2003.50032

Diabetes, Metabolic Syndrome and Obesity: Targets and Therapy

\section{Publish your work in this journal}

Diabetes, Metabolic Syndrome and Obesity: Targets and Therapy is an international, peer-reviewed open-access journal committed to the rapid publication of the latest laboratory and clinical findings in the fields of diabetes, metabolic syndrome and obesity research. Original research, review, case reports, hypothesis formation, expert opinion and commentaries are all considered for publication. The manuscript management system is completely online and includes a very quick and fair peer-review system, which is all easy to use. Visit http://www.dovepress.com/testimonials.php to read real quotes from published authors. 